\title{
Integrated analysis of pseudogene RP11-564D11.3 expression and its potential roles in hepatocellular carcinoma
}

\author{
Hui Song ${ }^{\ddagger} 1,2$, Jue Yang ${ }^{\ddagger}, 3,4$, Yongqiang Zhang ${ }^{5}$, Jianjiang Zhou*,1,2, Yanmei Li**,3,4 \& \\ Xiaojiang Hao***,3,4 \\ ${ }^{1}$ The Key Laboratory of Endemic \& Ethnic Diseases, Guizhou Medical University, Ministry of Education, Guiyang 550004, PR China \\ ${ }^{2}$ The Key Laboratory of Medical Molecular Biology, Guizhou Medical University, Guizhou Province, Guiyang 550004, PR China \\ ${ }^{3}$ The State Key Laboratory of Functions \& Applications of Medicinal Plants, Guizhou Medical University, Guiyang 550014, PR China \\ ${ }^{4}$ The Key Laboratory of Chemistry for Natural Products of Guizhou Province \& Chinese Academic of Sciences, Guiyang 550014, PR \\ China \\ ${ }^{5}$ School of Pharmaceutical Sciences, Guizhou University, Guiyang, 550025, PR China \\ *Author for correspondence: jianjiangzhou@sina.cn \\ **Author for correspondence: liyanmei518@hotmail.com \\ ***Author for correspondence: haoxj2013@126.com \\ ${ }^{\ddagger}$ Authors contributed equally
}

\begin{abstract}
Aim: We aim to identify differentially expressed pseudogenes and investigate their functional roles in carcinogenesis. Materials \& methods: Here, we identify dysregulated pseudogenes, analyze their prognostic values and investigate their potential functions through pseudogene-miRNA-mRNA network from public -omics repositories. Results: We identified 16 frequently upregulated pseudogenes among which high expression levels of RP11-564D11.3 was significantly associated with poor overall survival in seven types of human cancers. RP11-564D11.3 was strongly correlated with pathways in cancer, PI3K-Akt signaling pathway and the neurotrophin signaling pathway. Further studies revealed that RP11-564D11.3 functions as a competitive endogenous RNA through targeting VEGFA in hepatocellular carcinoma. Conclusion: Our findings suggest $R P 11-564 D 11.3$ as a novel biomarker and therapeutic potential target against hepatocellular carcinoma.
\end{abstract}

First draft submitted: 17 September 2018; Accepted for publication: 10 October 2018; Published online: 26 October 2018

Keywords: hepatocellular carcinoma • pseudogene • RP11-564D11.3 • VEGFA

Global transcriptomic analysis have shown that of the $70-90 \%$ of human genome transcribed into RNAs, only $1-2 \%$ is protein-coding genes and the remainder is noncoding RNA (ncRNA) [1]. To date, several ncRNAs have been identified, such as miRNA, long ncRNAs, and circular RNAs, as well as pseudogenes. Emerging evidence have established that dysregulated ncRNAs play crucial roles in physiological and pathophysiological processes, including control of chromosome modification, RNA processing, translation inhibition and mRNA degradation [2-4].

The competitive endogenous RNA (ceRNA) hypothesis may explain, at least in part, the complex biological functions of ncRNA in the pathogenesis of human cancer [5,6]. The ceRNA crosstalk is based on an 'miRNA response element language' in which any transcripts containing the same miRNA response element can co-regulate each other by competing for binding to shared miRNAs [6]. The ceRNA network was discovered in diverse species, including humans, mice, plants and viruses [7-10]. Therefore, the ceRNA hypothesis provides a basic framework to predict the potential functions of novel ncRNAs.

Pseudogenes are a class of ncRNAs and defunct copies of protein-coding genes arisen from random replication and mutation during evolutionary process [11,12]. There are two types of pseudogenes: processed and nonprocessed, which were generated from gene retrotransposition and duplication events, respectively [13,14]. Recent advances have shown that pseudogenes can regulate their parental genes and also unrelated genes in multiple ways in the pathogenesis of human cancer [15-17]. For example, pseudogene PTENP1 functions as a ceRNA to regulate parental PTEN expression and affect tumorigenesis by sponging miR-19b, miR-21 and miR-499-5p [18-20]. Meanwhile,

Future Medicine 
PTENP1 can also act as ceRNA for PTEN-independent genes, such as SOCS6, PHLPP and P62 [21,22]. These findings suggest that pseudogenes can exert biological functions through regulating not only their parental genes but also unrelated genes.

In this study, we first identified differentially expressed pseudogenes by browsing the pseudogene database dreamBase. Then we explored the prognostic values of the dysregulated pseudogenes based on the Cancer Genome Atlas (TCGA) databases and further investigated its potential functions and molecular mechanisms through the pseudogene-miRNA-mRNA network.

\section{Materials \& methods}

Identification of differentially expressed pseudogenes

RNA-Seq expression data of pseudogenes in 32 types of human cancers were downloaded from the dreamBase database (http://rna.sysu.edu.cn/dreamBase/index.php) [23]. The cutoff for differentially expressed pseudogenes was set at $\mid \log 2 \mathrm{FCl}>2.0$. Their expression levels were further validated by expression landscape. Heat map of differentially expressed pseudogenes was visualized using MeV v4.8.1 [24].

\section{Kaplan-Meier survival analysis}

For pseudogenes, Kaplan-Meier overall survival analysis and log-rank test were used to evaluate the statistical significance of survival differences between the two groups using GEPIA [25]. The cut-off value between the two groups was 'Median'. For mRNA, Kaplan-Meier overall survival analysis was performed using Kaplan-Meier Plotter [26].

\section{Construction of pseudogene-miRNA-mRNA regulatory network}

miRNAs binding to pseudogene were identified using starBase v2.0 [27]. Target genes for identified miRNA were retrieved from miRTarBase [28]. Pseudogene-miRNA-mRNA ceRNA network were visualized using Cytoscape v3.4.0 [29].

\section{Gene ontology \& KEGG pathway enrichment analysis}

Pathway enrichment analysis for enriched target genes was conducted using ClueGO v2.3.5 plugin of Cytoscape v3.4.0 [30]. GO items/pathways with $\mathrm{p}<0.05$ were considered significant difference.

\section{Protein-protein interaction network \& hub genes analysis}

Protein-protein interaction network for enriched target genes was analyzed using STRING v10.0 and visualized by Cytoscape v3.4.0 [31]. Genes with top ten degree were identified as hub genes using Centiscape 2.2 plugin of Cytoscape v3.4.0 [32].

\section{Gene expression analysis}

VEGFA mRNA and protein expression levels in hepatocellular carcinoma tissues were analyzed using ULACAN and HumanProteinAtlas, respectively [33,34].

\section{Mutations \& copy-number alterations analysis}

DNA mutations and copy-number alterations of hub genes from 442 patients with liver hepatocellular carcinoma (TCGA, provisional) were analyzed using cBioPortal [35].

\section{Results}

Identification of dysregulated pseudogenes in human cancers

In China, lung adenocarcinoma, stomach adenocarcinoma and liver adenocarcinoma are the most common malignant tumors with high morbidity and mortality. To investigate the potential roles of pseudogenes in human cancers, we first identified frequently abnormally expressed pseudogenes using dreamBase. As shown in Figure 1A $\&$ B, 42 upregulated and two downregulated pseudogenes were identified in lung adenocarcinoma, stomach adenocarcinoma and hepatocellular carcinoma. After screening by pseudogene names and expression levels, 16 frequently upregulated pseudogenes in 32 types of human cancers were selected for further investigation (Table 1). Their expression levels in 32 types of human cancers were shown in Figure 1C. These results indicate that these 16 upregulated pseudogenes may play key roles in carcinogenesis. 


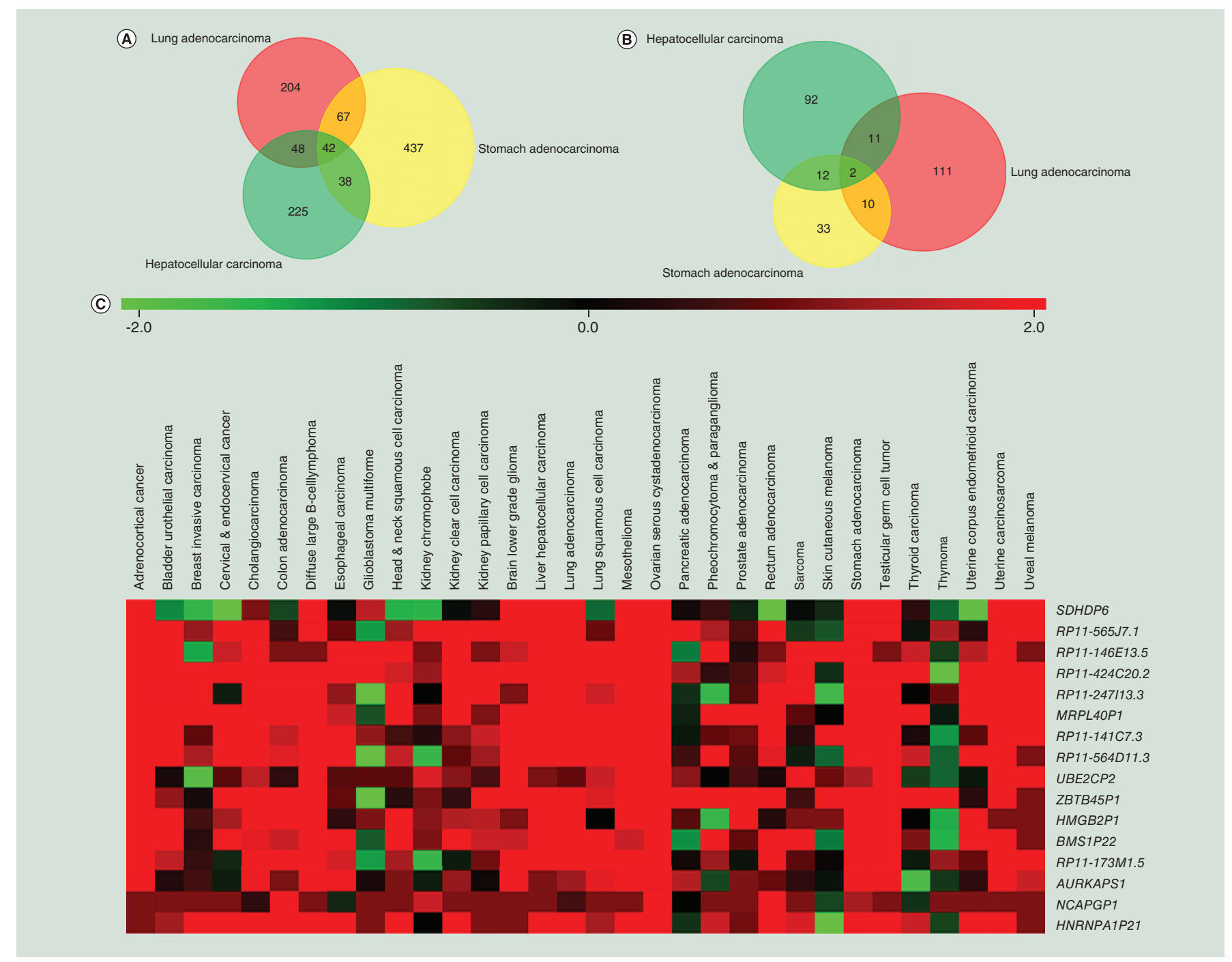

Figure 1. Identification of differentially expressed pseudogenes in human cancers. RNA-Seq expression data of pseudogenes in 32 types of human cancers were downloaded from dreamBase (http://rna.sysu.edu.cn/dreamBase/index.php). The cutoff for differentially expressed pseudogenes was set at $|\log 2 \mathrm{FC}|>2.0$. Their expression levels were further validated by expression landscape. (A) Upregulated pseudogenes in lung adenocarcinoma, stomach adenocarcinoma and hepatocellular carcinoma. (B) Downregulated pseudogenes in lung adenocarcinoma, stomach adenocarcinoma and hepatocellular carcinoma. (C) Heat map of 16 frequently upregulated pseudogenes in 32 types of human cancers visualized by MeV v4.8.1. Red represents upregulated genes and green represents downregulated genes.

\section{Prognostic values of differentially expressed pseudogenes}

Next, we explored the prognostic values of 16 dysregulated pseudogenes in human cancers based on TCGA data using GEPIA. As shown in Figure 2, high expression levels of RP11-424C20.2 were identified to be most significantly correlated with overall survival, worse prognosis in seven human cancers and better prognosis in thymoma. High expression levels of RP11-564D11.3 was associated with worse outcomes in seven human cancers. High mRNA expression levels of SDHDP6 was significantly associated with better outcome in patients with breast invasive carcinoma ( $\mathrm{p}=0.032)$. Increased RP11-146E13.5, ZBTB45P1 and HMGB2P1 were significantly associated with poor outcomes in pheochromocytoma and paraganglioma $(\mathrm{p}=0.0094)$, cervical and endocervical cancer $(\mathrm{p}=0.028)$ and kidney clear cell carcinoma $(\mathrm{p}=0.0053)$, respectively. These results indicate that RP11-564D11.3 may have high prognostic values, and function as an oncogene in tumorigenesis and tumor progression. 
Table 1. Information of 16 frequently upregulated pseudogenes in human cancers identified by dreamBase.

\begin{tabular}{|lllll|}
\hline $\begin{array}{l}\text { Pseudogene } \\
\text { name }\end{array}$ & Ensembl ID & Genome location & Gene type & Parent gene name \\
\hline SDHDP6 & ENST00000414693 & chr1:25294164-25294643 & processed_pseudogene & SDHD \\
\hline RP11-565J7.1 & ENST00000416056 & chr1:211936249-211936634 & processed_pseudogene & PIN4 \\
\hline RP11-146E13.5 & ENST00000619332 & chr14:19131842-19132890 & processed_pseudogene & TOMM40 \\
\hline RP11-424C20.2 & ENST00000540175 & chr12:20551590-20553012 & processed_pseudogene & UHRF1 \\
\hline RP11-247113.3 & ENST00000445477 & chr22:31559483-31559939 & processed_pseudogene & RPS18 \\
\hline MRPL4OP1 & ENST00000542687 & chr12:67351436-67352039 & processed_pseudogene & MRPL40 \\
\hline RP11-141C7.3 & ENST00000603917 & chr3:195650146-195651472 & processed_pseudogene & PPP4R2 \\
\hline RP11-564D11.3 & ENST00000368895 & chr10:122879851-122898024 & transcribed_processed_pseudogene & C10orf88 \\
\hline UBE2CP2 & ENST00000583290 & chr18:22900486-22900995 & processed_pseudogene & UBE2C \\
\hline ZBTB45P1 & ENST00000452761 & chr2:109986939-109988394 & processed_pseudogene & ZBTB45 \\
\hline HMGB2P1 & ENST00000591146 & chr19:1203209-1203842 & transcribed_processed_pseudogene & HMGB2 \\
\hline BMS1P22 & ENST00000414726 & chr22:15805847-15806503 & transcribed_unprocessed_pseudogene & BMS1 \\
\hline RP11-173M1.5 & ENST00000582878 & chr17:27351858-27352584 & processed_pseudogene & SYPL1 \\
\hline AURKAPS1 & ENST00000451805 & chr1:220266706-220267917 & transcribed_processed_pseudogene & AURKA \\
\hline NCAPGP1 & ENST00000505112 & chr8:120635558-120638204 & processed_pseudogene & NCAPG \\
\hline HNRNPA1P21 & ENST00000424115 & chr3:39334979-39335939 & processed_pseudogene & HNRNPA1 \\
\hline
\end{tabular}

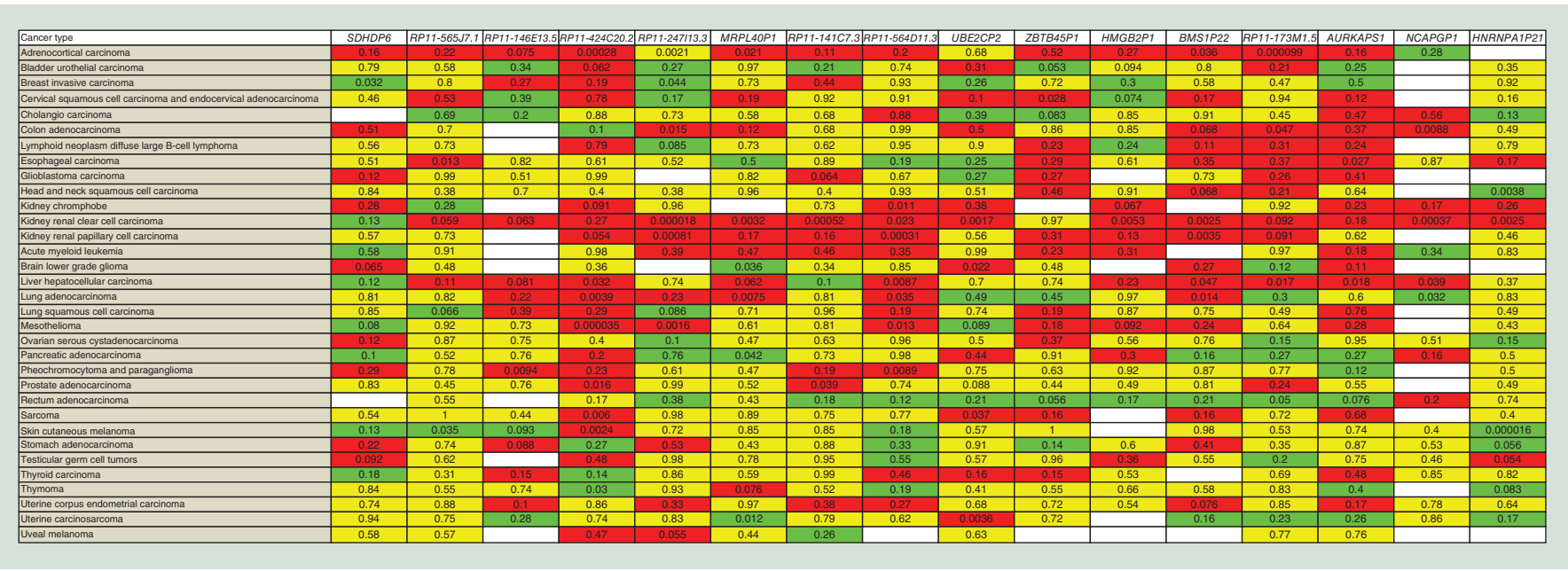

Figure 2. Prognostic values of 16 differentially expressed pseudogenes in 33 types of human cancers. Kaplan-Meier overall survival analysis for 16 frequently upregulated pseudogenes were performed using GEPIA. The cut-off value between the two groups was 'Median'. GEPIA uses log-rank test, a.k.a the Mantel-Cox test, for hypothesis test. p value $<0.05$ was considered statistically significant. Red represents poor outcome, yellow represents neutral outcome, green represents good outcome and white means that the sample size is insufficient at custom thresholds.

GEPIA: Gene expression profiling interactive analysis.

Pseudogene-miRNA-mRNA regulatory network

ceRNA is an important regulatory mechanism for pseudogenes, in which pseudogenes function as RNA sponges for miRNAs and regulate target genes' expression through competing for binding of common miRNAs. starBase was used to identify potential miRNAs which can bind to pseudogenes. For RP11-564D11.3, miR-9-5p, miR-101-3p and the miR-200 family (miR-141-3p, miR-200a-3p, miR-200b-3p, miR-200c-3p and miR-429) were identified to be candidate miRNAs (Table 2). miRNA target genes were validated by at least one strongly experimental method (reporter assay, Western blot or qRT-PCR) using miRTarBase. In total, 338 genes were identified to be direct targets of candidate miRNAs and the pseudogene-miRNA-mRNA regulatory network was then visualized using Cytoscape (Figure 3). Thus, RP11-564D11.3 may exert biological functions through regulating these target genes. 


\begin{tabular}{|c|c|c|c|c|}
\hline miRNA names & mirAccession & TargetSites & BioComplex & ClipReadNum \\
\hline miR-200b-3p & MIMAT0000318 & 1 & 2 & 458 \\
\hline miR-200a-3p & MIMAT0000682 & 1 & 6 & 709 \\
\hline miR-429 & MIMAT0001536 & 1 & 2 & 458 \\
\hline miR-101-3p & MIMAT0000099 & 1 & 6 & 709 \\
\hline miR-9-5p & MIMAT0000441 & 1 & 1 & 3 \\
\hline $\operatorname{miR}-200 c-3 p$ & MIMAT0000617 & 1 & 2 & 458 \\
\hline miR-141-3p & MIMAT0000432 & 1 & 6 & 709 \\
\hline
\end{tabular}

\begin{tabular}{|lllll|}
\hline \multicolumn{5}{l}{ Table 3. Ten hub genes identified by Centiscape 2.2. } \\
\hline Gene names & Degree unDir & Closeness unDir & Betweenness unDir & Gene descriptions \\
\hline TP53 & 150 & 0.002155 & $10,580.06$ & Tumor protein p53 \\
\hline MYC & 123 & 0.002024 & 4377.777 & MYC proto-oncogene, bHLH transcription factor \\
\hline VEGFA & 112 & 0.001992 & 4076.718 & Vascular endothelial growth factor A \\
\hline EGFR & 109 & 0.001949 & 5191.221 & Epidermal growth factor receptor \\
\hline JUN & 107 & 0.001953 & 2706.145 & Jun proto-oncogene, AP-1 transcription factor subunit \\
\hline CTNNB1 & 101 & 0.001938 & 3890.535 & Catenin beta 1 \\
\hline NOTCH1 & 99 & 0.001942 & 2583.603 & Notch 1 \\
\hline IL6 & 97 & 0.001894 & 2853.689 & Interleukin 6 \\
\hline CCND1 & 96 & 0.001905 & 2476.921 & Cyclin D1 \\
\hline BCL2 & 94 & 0.001887 & 1533.52 & B cell leukemia/lymphoma 2 \\
\hline
\end{tabular}

\section{Functional enrichment analysis of miRNA target genes}

To investigate the potential functions of RP11-564D11.3 in cancer development, we performed GO and KEGG pathway enrichment analysis using analyzed ClueGo. For RP11-564D11.3, $81 \mathrm{GO}$ terms were enriched and finally classified into 24 groups with KappaScore (Figure 4). The most significantly enriched gene sets were pathways in cancer (KEGG: 05200; $\mathrm{p}=8.84 \mathrm{E}-28)$, the PI3K-Akt signaling pathway (KEGG: 04151; $\mathrm{p}=1.13 \mathrm{E}-17$ ) and neurotrophin signaling pathway (KEGG: 04722; $\mathrm{p}=9.01 \mathrm{E}-11$ ). Consistent with the prognostic value of RP11$564 D 11.3$ in human cancers, renal cell carcinoma, small-cell lung cancer, non-small-cell lung cancer, hepatitis $\mathrm{C}$ and hepatitis B were enriched. These findings confirm that upregulated RP11-564D11.3 is involved in the initiation and progression of hepatocellular carcinoma, lung adenocarcinoma and renal cell carcinoma.

\section{VEGFA was identified as target gene of RP11-564D11.3}

To further analyze the functional roles of RP11-564D11.3, a protein-protein interaction network was generated using STRING and hub genes were selected using Centiscape 2.2 (Figure 5). Genes with top ten degree (TP53, MYC, VEGFA, EGFR, JUN, CTNNB1, NOTCH1, IL6, CCND1 and BCL2) were identified as hub genes for RP11-564D11.3 (Table 3). Pearson correlation analysis between RP11-564D11.3 and ten hub genes in kidney renal clear cell carcinoma, hepatocellular carcinoma and lung adenocarcinoma results showed that six out of ten hubs (TP53, VEGFA, JUN, CTNNB1, NOTCH1 and BCL2) in hepatocellular carcinoma, three out of ten hubs (VEGFA, IL6 and BCL2) in lung adenocarcinoma and one out of ten hubs (EGFR) in kidney renal clear cell carcinoma were significantly correlated with RP11-564D11.3 expression levels, respectively (Table 4). These results indicate that RP11-564D11.3 may possess higher prognostic values or play more crucial roles in hepatocellular carcinoma compared with that of kidney renal clear cell carcinoma and lung adenocarcinoma.

Then we investigated the gene mutations and copy-number alterations of these ten hubs in hepatocellular carcinoma using cBioportal. The results demonstrated that TP53 and CTNNB1 were exclusively mutant, occurred in $111(30.8 \%)$ of 360 patients and $96(26.7 \%)$ of 360 patients, respectively. MYC, VEGFA and CCND1 were significantly amplificated, occurred in $63(17.5 \%)$ of 360 patients, $30(8.3 \%)$ of 360 patients and $26(7.2 \%)$ of 360 patients, respectively (Figure 6). We also analyzed the prognostic values of ten hubs in hepatocellular carcinoma using Kaplan-Meier plotter from GEO datasets. We found that high mRNA expression levels of TP53, $E G F R$ and CCND1 were significantly associated with good overall survival $(\mathrm{p}=0.029 ; \mathrm{p}=0.0085 ; \mathrm{p}=0.03$, 


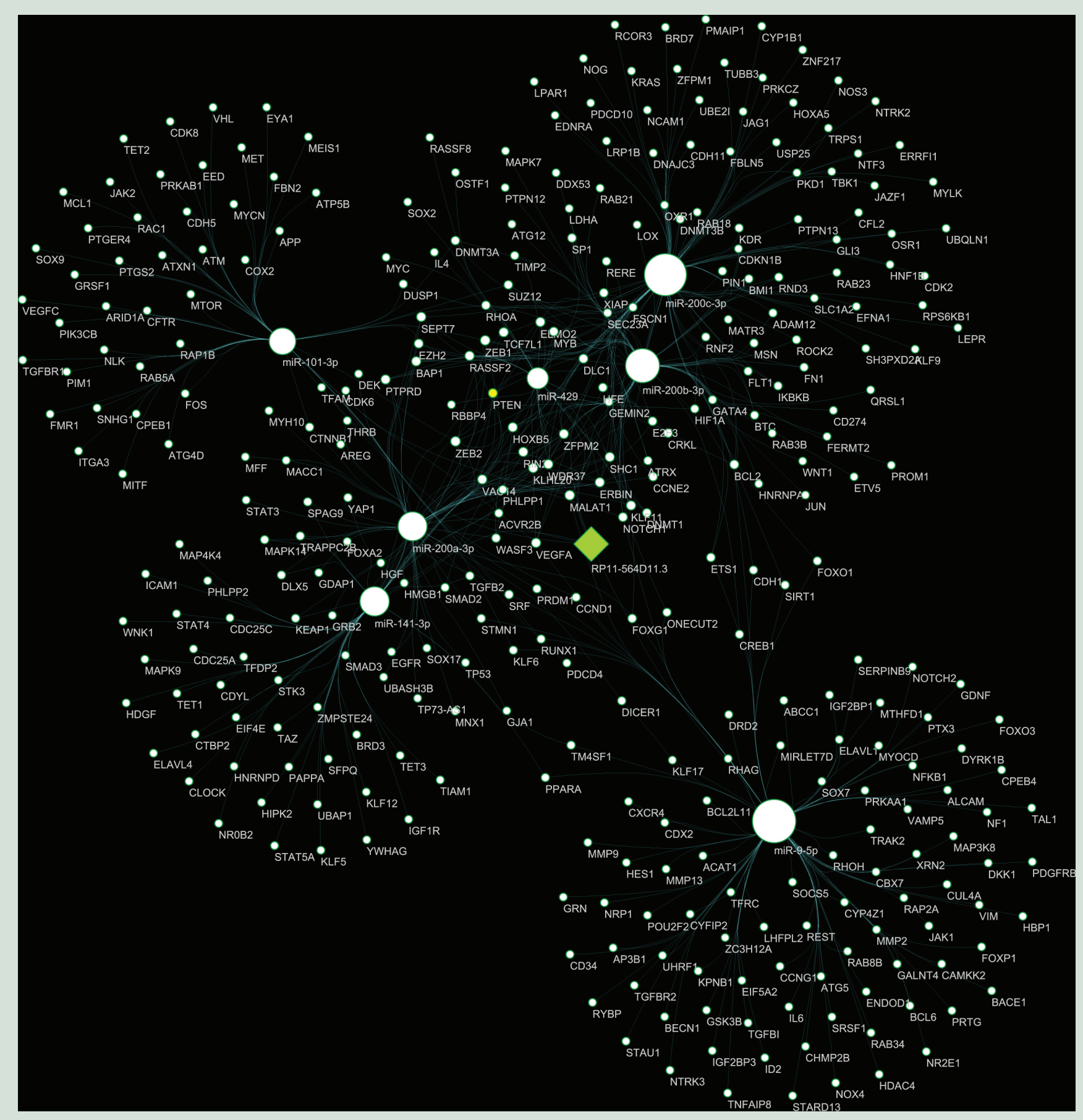

Figure 3. Construction of pseudogene-miRNA-mRNA regulatory network. miR-141-3p, miR-200a-3p, miR-200b-3p, miR-200c-3p and miR-429 were identified as candidate miRNAs targeting RP11-564D11.3 using starBase v2.0. Target genes for these miRNAs were retrieved by at least one of strongly experimental methods (reporter assay, Western blot or qRT-PCR) from miRtarbase. Pseudogene-miRNA-mRNA interaction network was visualized using Cytoscape v3.4.0.

respectively; Table 5). Only high mRNA expression level of VEGFA was significantly associated with poor overall survival $(\mathrm{p}=0.0025)$. According to ceRNA hypothesis, VEGFA might be a potential target of RP11-564D11.3 in hepatocellular carcinoma (Figure 7A). VEGFA mRNA and protein expression levels were further validated higher 


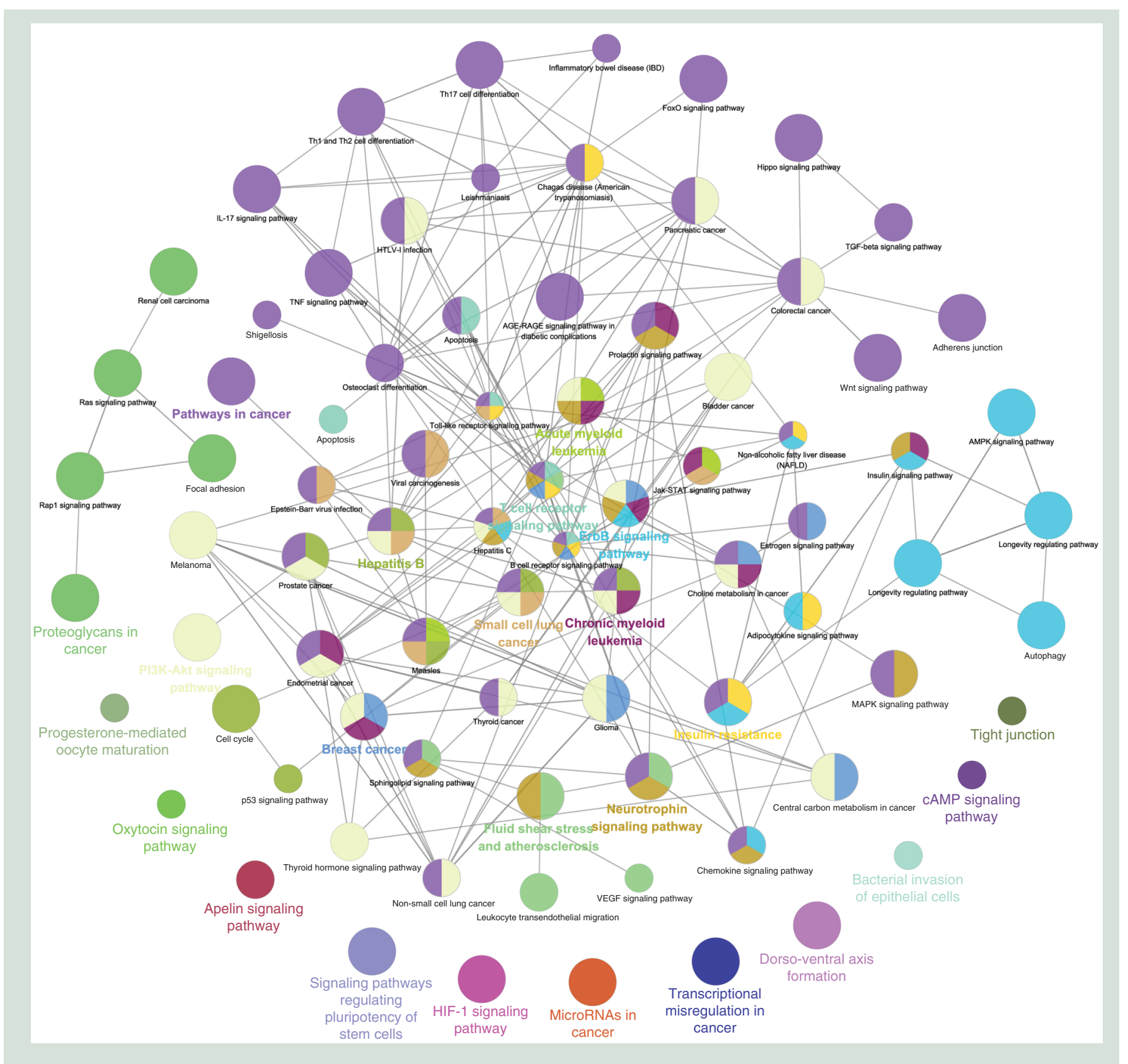

Figure 4. Functional enrichment analysis of miRNA target genes. GO and KEGG pathway enrichment analysis of 338 RP11-564D11.3-related genes were performed using ClueGo v2.3.5 plugin of Cytoscape v3.4.0. Total 81 terms/pathways were enriched ( $p$ $<0.05$ ). After merging redundant groups with $>50.0 \%$ overlap, 24 terms/pathways were finally selected. Enrichment $/$ depletion (two-sided hypergeometric test) and Bonferroni step down were used for statistical test and correction method, respectively. Pathways in cancer (KEGG: 05200; $p=8.84 \mathrm{E}-28$ ), PI3K-Akt signaling pathway (KEGG: 04151; $p=1.13 \mathrm{E}-17$ ) and Neurotrophin signaling pathway (KEGG: 04722; $\mathrm{p}=9.01 \mathrm{E}-11$ ) were the top three significantly enriched gene sets.

in hepatocellular carcinoma tissues compared that of normal tissues (Figure $7 \mathrm{~B} \& \mathrm{C}$ ). These finding indicate that RP11-564D11.3 functions as a ceRNA through targeting VEGFA in hepatocellular carcinoma.

\section{Discussion}

Pseudogenes have long been dismissed as 'junk' or 'fossil' DNA. Recent studies have characterized several functional pseudogenes, such as PDIA3P1, NMRAL2P and HMGA1Ps [36-38]. HMGA1P6 and HMGA1P7 are two processed pseudogenes, and overexpression of HMGA1PG and HMGA1P7 could increase the levels of HMGA1 and other 


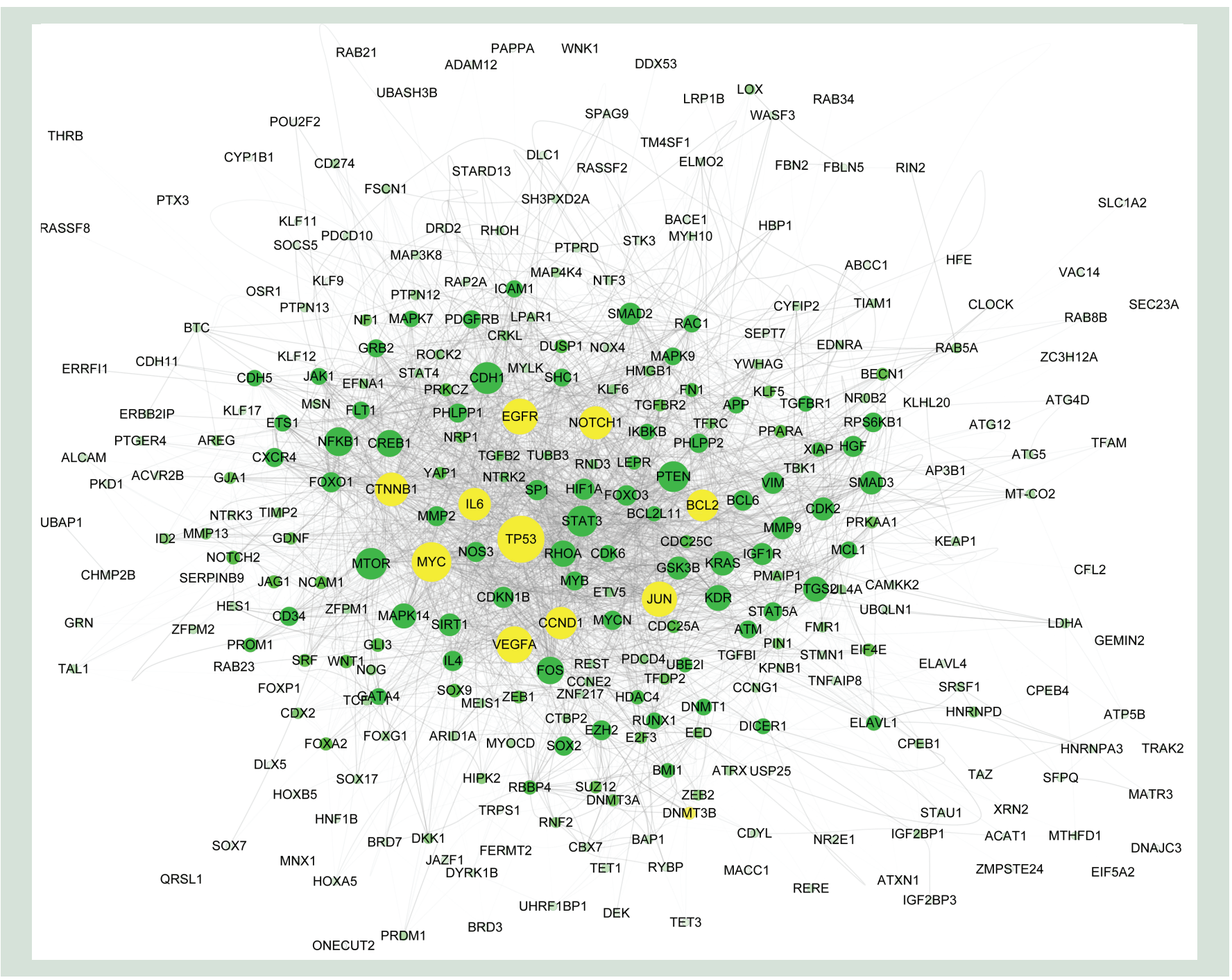

Figure 5. Identification of hub genes. The protein-protein interaction network of 338 genes was constructed using STRING v10.0. Confidence score $\geq 0.4$ was set as the cutoff criterion. Then the gene degree was further analyzed by Centiscape 2.2 plugin of Cytoscape v3.4.0. Genes with top 10 degree value were identified as hub genes.

\begin{tabular}{|c|c|c|c|c|c|c|}
\hline Gene names & \multicolumn{2}{|c|}{ Hepatocellular carcinoma } & \multicolumn{2}{|c|}{ Lung adenocarcinoma } & \multicolumn{2}{|c|}{ Kidney renal clear cell carcinoma } \\
\hline TP53 & 0.015 & 0.13 & 0.059 & 0.2 & 0.58 & 0.025 \\
\hline MYC & 0.39 & 0.045 & 0.067 & 0.084 & 0.35 & 0.041 \\
\hline VEGFA & $1.90 \mathrm{E}-07$ & 0.27 & 0.0031 & 0.13 & 0.22 & -0.053 \\
\hline JUN & 0.011 & 0.13 & 0.055 & -0.087 & 0.83 & -0.0097 \\
\hline CTNNB1 & 0.0012 & 0.17 & 0.096 & 0.076 & 0.48 & -0.031 \\
\hline NOTCH1 & $5.10 \mathrm{E}-08$ & 0.28 & 0.23 & -0.054 & 0.37 & -0.039 \\
\hline IL6 & 0.91 & 0.0056 & 0.01 & 0.12 & 0.46 & 0.032 \\
\hline CCND1 & 0.77 & 0.015 & 0.13 & 0.07 & 0.19 & -0.057 \\
\hline
\end{tabular}




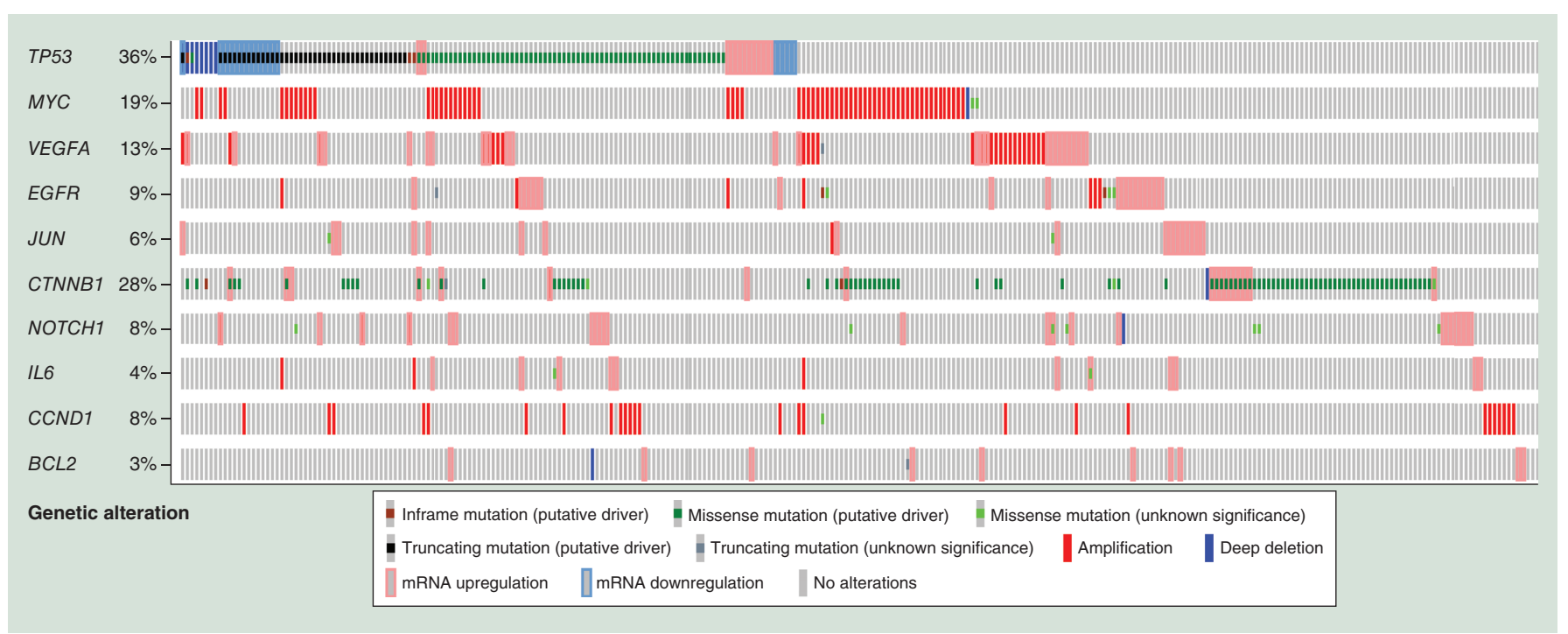

Figure 6. Gene mutations and copy-number alterations analysis of ten hub genes in $\mathbf{4 4 2}$ patients (only $\mathbf{3 6 0}$ patients' data available) with liver hepatocellular carcinoma (TCGA, provisional) were analyzed using cBioPortal. Tumor samples are shown in columns. TCGA: The Cancer Genome Atlas.

Table 5. Prognostic values of ten hub genes in hepatocellular carcinoma identified by Kaplan-Meier plotter.

\begin{tabular}{|lllll|}
\hline Gene names & RNAseq IDs & p-value & HRs with 95\% Cls & Prognostic outcomes \\
\hline TP53 & 7157 & 0.029 & $0.65(0.45-0.96)$ & Good \\
\hline MYC & 4609 & 0.088 & $1.38(0.95-1.99)$ & Poor \\
\hline VEGFA & 7422 & 0.0025 & $1.74(1.21-2.5)$ & Poor \\
\hline EGFR & 1956 & 0.0085 & $0.61(0.43-0.89)$ & Good \\
\hline JUN & 3725 & 0.36 & $1.18(0.83-1.66)$ & Poor \\
\hline CTNNB1 & 1499 & 0.37 & $1.17(0.83-1.65)$ & Poor \\
\hline NOTCH1 & 4851 & 0.10 & $0.75(0.53-1.06)$ & Good \\
\hline IL6 & 3569 & 0.42 & $1.16(0.81-1.65)$ & Poor \\
\hline CCND1 & 595 & 0.03 & $0.68(0.48-0.97)$ & Good \\
\hline BCL2 & 596 & 0.072 & $0.72(0.51-1.03)$ & Good \\
\hline CI CONDF
\end{tabular}

$\mathrm{Cl}$ : Confidence interval; HR: Hazard ratio.

cancer-related proteins through a ceRNA mechanism, and contribute to carcinogenesis [38-41]. However, pseudogenes and their underlying regulatory mechanisms remain largely unknown. Identifying differentially expressed pseudogenes and exploring their potential functions will facilitate the prognostic prediction and targeted therapy of human cancers.

In this study, we explored the abnormally expressed pseudogenes in hepatocellular carcinoma, lung adenocarcinoma and stomach adenocarcinoma by utilizing the dreamBase database and identified 16 frequently upregulated pseudogenes in 32 types of human cancers. Kaplan-Meier survival analysis revealed that all the 16 pseudogenes were significantly associated with overall survival in at least one type of human cancer. Among them, high expression levels of RP11-564D11.3 were obviously correlated with poor overall survival of patients with kidney diseases, hepatocellular carcinoma, lung adenocarcinoma, mesothelioma and pheochromocytoma and paraganglioma. These findings suggest that RP11-564D11.3 may function as an oncogene involved in carcinogenesis.

ceRNAs hypothesis provides an important predictive pattern for unidentified ncRNA. In this pattern, pseudogenes act as decoys for miRNAs to affect cognate or noncognate target genes. To predict the functional roles of RP11-564D11.3, we constructed a pseudogene-miRNA-mRNA interaction network. RP11-564D11.3 was identified to be a direct target gene for miR-9-5p, miR-101-3p and the miR-200 family, and 334 genes may be potential regulatory targets of RP11-564D11.3. 


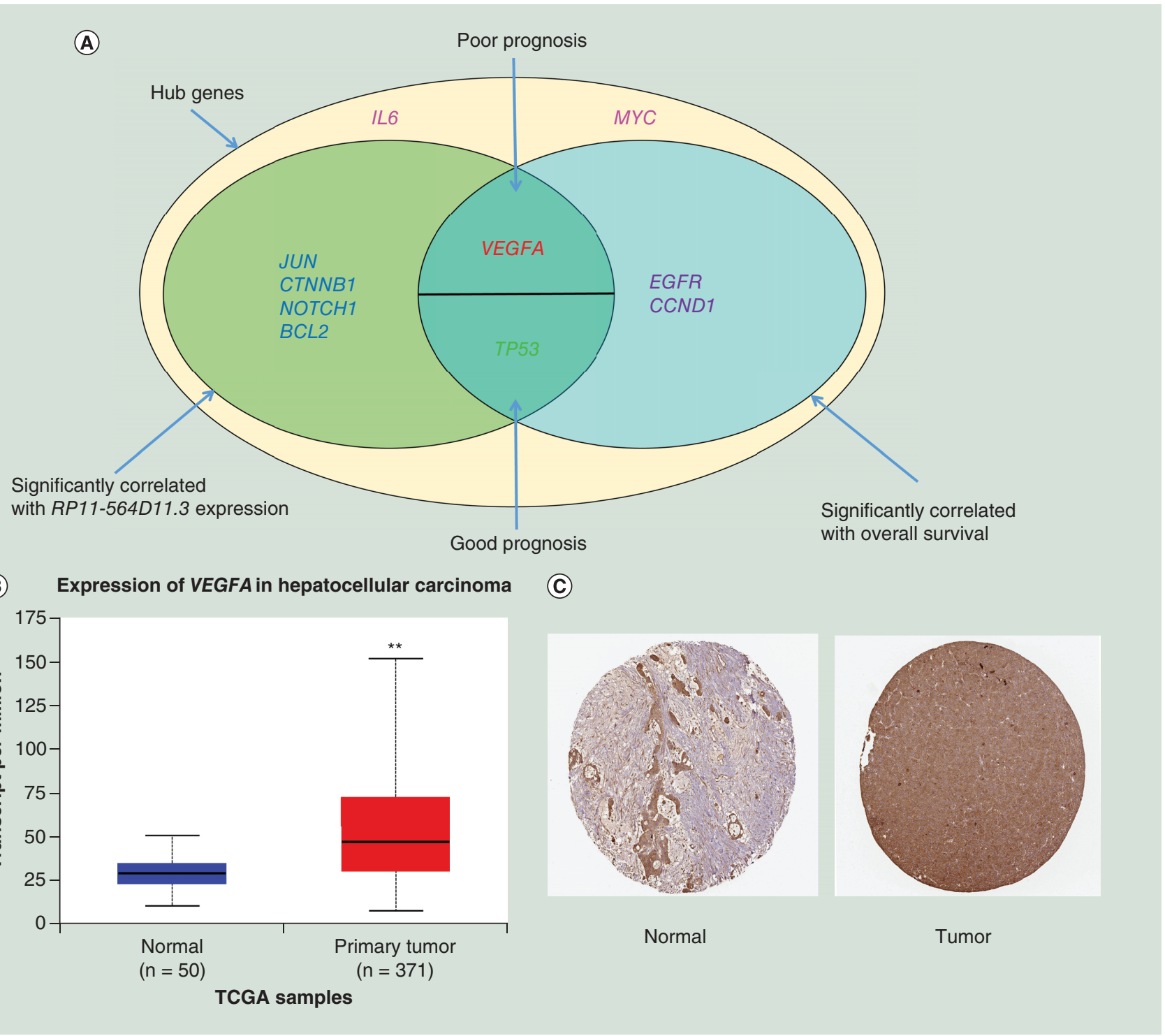

Figure 7. VEGFA was identified as the target gene of RP11-564D11.3. (A) Pearson correlation analysis and prognostic outcomes analysis of 10 hub genes revealed VEGFA was a target gene of RP11-564D11.3. (B) VEGFA mRNA expression level in 371 hepatocellular carcinoma tissues and 50 normal hepatocellular tissues was analyzed using UALCAN. (C) VEGFA protein expression level in hepatocellular carcinoma tissues and normal hepatocellular tissues was analyzed using immunohistochemical staining from HumanProteinAtlas.

** $p$ value $<0.01$

TCGA: The Cancer Genome Atlas.

Several studies demonstrated that miR-9-5p has a dual role in carcinogenesis as either a tumor suppressor or a tumor promoter. For example, upregulated miR-9-5p promotes cell growth and metastasis of non-small-cell lung cancer and hepatocellular carcinoma [42,43]. On the other hand, miR-9-5p inhibited cell proliferation and progression of gastric cancer and ovarian cancer [44,45]. miR-101-3p was frequently downregulated and could regulate growth, metastasis and tumor metabolism in a variety of human cancers, including bladder cancer, hepatocellular carcinoma, lung squamous carcinoma and breast cancer [46-49]. The miR-200 family was reported as tumor suppressor miRNA and has been implicated in epithelial-to-mesenchymal transition, metastasis, angiogenesis and chemoresistance in many different cancers [50-53].

Functional enrichment analysis of 334 target genes revealed multiple RP11-564D11.3-associated GO items/pathways. We also observed that pathways in cancer (KEGG: 05200), PI3K-Akt signaling pathway (KEGG: 04151) and neurotrophin signaling pathway (KEGG: 04722) were the top three significantly enriched gene sets. 
The PI3K-Akt signaling pathway is one of the most frequently activated signal transduction pathways in human cancer. Activation of the PI3K-Akt signaling pathway triggers a cascade of responses, from cell proliferation and survival to chemoresistance [54-56]. Interestingly, we found that RP11-564D11.3 was also significantly associated with neurotrophin signaling pathway. The neurotrophins family comprises four related proteins; NGFB, BDNF, NTF3 and NTF4/5. They play important roles in proliferation, differentiation and survival of neural cells [54]. Recent studies revealed neurotrophins and their receptors are also expressed in cancer cells and regulate cell proliferation, aggressiveness and metastasis [58-60].

In this study, we also performed hub genes identification and person correlation analysis between $R P 11-$ $564 D 11.3$ and hub genes. We observed mRNA expression levels of six out of ten hubs (TP53, VEGFA, JUN, CTNNB1, NOTCH1 and BCL2) were significant positively correlated with RP11-564D11.3 expression level in hepatocellular carcinoma, indicating that RP11-564D11.3 may play more crucial roles in the hepatocellular carcinoma pathogenesis. Prognostic values analysis of hepatocellular carcinoma patients from GEO datasets showed TP53, VEGFA, EGFR and CCND1 were significantly associated with overall survival of hepatocellular carcinoma patients. Mutations and copy-number alterations analysis revealed that TP53, CTNNB1, MYC, VEGFA and $C C N D 1$ were exclusively mutant or amplified in hepatocellular carcinoma. These findings suggest that $V E G F A$ was more likely to be potential target gene for RP11-564D11.3. Several studies demonstrated VEGFA is a well-known angiogenesis inducer, and overexpression of VEGFA can inhibit hepatocellular carcinoma cell proliferation, invasion and angiogenesis [61-63]. Further investigations are needed to confirm the RP11-564D11.3-mediated ceRNA regulatory network and explore the functional roles of RP11-564D11.3 in the tumorigenesis of hepatocellular carcinoma.

\section{Conclusion}

In summary, the present study for the first time systematically revealed that pseudogene RP11-564D11.3 was frequently upregulated and associated with poor prognosis in hepatocellular carcinoma. Our findings also indicated that RP11-564D11.3 may function as an oncogene or ceRNA through targeting VEGFA and its potential roles in carcinogenesis is worthy of further study. More importantly, we here provide a reliable prediction method for unidentified genes based on a series of analysis, including prognostic values evaluation, gene-miRNA-mRNA ceRNA network, hub genes recognition and expression correlation analysis.

\section{Future perspective}

Pseudogenes play a pivotal and multifaceted role in human cancer and RP11-564D11.3 can serve as a useful biomarker and novel therapeutic target against hepatocellular carcinoma.

\section{Summary points}

- We identified 16 frequently upregulated pseudogenes in human cancers using dreamBase.

- We found that high expression level of RP11-564D11.3 was significantly associated with poor overall survival in seven types of human cancers.

- Through pseudogene-miRNA-mRNA network, we identified seven candidate miRNAs and 338 RP11-564D11.3-related target genes.

- Functional enrichment analysis revealed that RP11-564D11.3 was strongly correlated with pathways in cancer, such as the PI3K-Akt signaling pathway and the neurotrophin signaling pathway.

- We demonstrated that RP11-564D11.3 functions as a competitive endogenous RNA or oncogene through targeting VEGFA in hepatocellular carcinoma.

- We provided a reliable prediction method for unidentified genes based on a series of bioinformatics analysis.

\section{Author contributions}

J Zhou, Y Li and X Hao conceived and designed the study; H Song, J Yang and Y Zhang obtained and analyzed data; H Song and J Yang drafted the manuscript; J Zhou and Y Li collected funds. All authors reviewed the manuscript before submission. All the authors approved the final version of the manuscript. 
Financial \& competing interests disclosure

This work was supported by grants from the National Natural Science Foundation of China (Numbers 31560326, 31760328, 81700169 and 81872772), Guizhou Science and Technology Project (Qiankehepintairencai [2017]5652), Guiyang Science and Technology Project (Zhuke[2017]5-16), the Natural Science Foundation of Guizhou Province (Number 201842920480710623), the "Light of the West" Talent Cultivation Program of the Chinese Academy of Sciences (Number 201684) and the Found for Guizhou Fourth Batch of "100 Talented Leaders". The authors have no other relevant affiliations or financial involvement with any organization or entity with a financial interest in or financial conflict with the subject matter or materials discussed in the manuscript apart from those disclosed.

No writing assistance was utilized in the production of this manuscript.

\section{Open access}

This work is licensed under theAttribution-NonCommercial-NoDerivatives 4.0 Unported License. To view a copy of this license, visit http://creativecommons.org/licenses/by-nc-nd/4.0/

\section{References}

Papers of special note have been highlighted as: $\bullet \bullet$ of considerable interest

1. ENCODE Project Consortium. An integrated encyclopedia of DNA elements in the human genome. Nature 489(7414), 57-74 (2012).

2. Kaikkonen MU, Adelman K. Emerging roles of non-coding RNA transcription. Trends Biochem. Sci. 43(9), 654-667 (2018).

3. Esteller M. Non-coding RNAs in human disease. Nat. Rev. Genet. 12(12), 861-874 (2011).

4. Beermann J, Piccoli MT, Viereck J et al. Non-coding RNAs in development and disease: background, mechanisms, and therapeutic approaches. Physiol. Rev. 96(4), 1297-1325 (2016).

5. Tay Y, Rinn J, Pandolf PP. The multilayered complexity of ceRNA crosstalk and competition. Nature 505(7483), 344-352 (2014).

6. Salmena L, Poliseno L, Tay Y et al. A ceRNA hypothesis: the Rosetta Stone of a hidden RNA language? Cell 146(3), 353-358 (2011).

7. Yang J, Li T, Gao C et al. FOXO1 $3^{\prime} \mathrm{UTR}$ functions as a ceRNA in repressing the metastases of breast cancer cells via regulating miRNA activity. FEBS Lett. 588(17), 3218-3224 (2014).

8. Grote P, Wittler L, Hendrix D et al. The tissue-specific lncRNA Fendrr is an essential regulator of heart and body wall development in the mouse. Dev. Cell. 24(2), 206-214 (2013).

9. Franco-Zorrilla JM, Valli A, Todesco M et al. Target mimicry provides a new mechanism for regulation of microRNA activity. Nat. Genet. 39(8), 1033-1037 (2007).

10. Cazalla D, Yario T, Steitz JA. Down-regulation of a host microRNA by a Herpesvirus saimiri noncoding RNA. Science 328(5985), 1563-1566 (2010).

11. Vanin EF. Processed pseudogenes. Characteristics and evolution. Biochim. Biophys. Acta. 782(3), 231-241 (1984).

12. Sasidharan R, Gerstein M. Genomics: protein fossils live on as RNA. Nature 453(7196), 729-731 (2008).

13. Esnault C, Maestre J, Heidmann T. Human LINE retrotransposons generate processed pseudogenes. Nat. Genet. 24(4), 363-367 (2000).

14. Balakirev ES, Ayala FJ. Pseudogenes: are they “junk” or functional DNA? Annu. Rev. Genet. 37, 123-151 (2003).

15. Wen YZ, Zheng LL, Qu LH et al. Pseudogenes are not pseudo any more. RNA Biol. 9(1), 27-32 (2012).

16. Poliseno L. Pseudogenes: newly discovered players in human cancer. Sci. Signal. 5(242), re5 (2012).

17. Pink RC, Wicks K, Caley DP et al. Pseudogenes: pseudo-functional or key regulators in health and disease? RNA 17(5), 792-798 (2011).

18. Li RK, Gao J, Guo LH et al. PTENP1 acts as a ceRNA to regulate PTEN by sponging miR-19b and explores the biological role of PTENP1 in breast cancer. Cancer Gene Ther. 24(7), 309-315 (2017).

19. Gao L, Ren W, Zhang L et al. PTENp1, a natural sponge of miR-21, mediates PTEN expression to inhibit the proliferation of oral squamous cell carcinoma. Mol. Carcinog. 56(4), 1322-1334 (2017).

20. Wang $\mathrm{L}$, Zhang N, Wang $\mathrm{Z}$ et al. Pseudogene PTENP1 functions as a competing endogenous RNA (ceRNA) to regulate PTEN expression by sponging miR-499-5p. Biochemistry (Mosc.) 81(7), 739-747 (2016).

21. Gong T, Zheng S, Huang S et al. PTENP1 inhibits the growth of esophageal squamous cell carcinoma by regulating SOCS6 expression and correlates with disease prognosis. Mol. Carcinog. 56(12), 2610-2619 (2017).

22. Chen CL, Tseng YW, Wu JC et al. Suppression of hepatocellular carcinoma by baculovirus-mediated expression of long non-coding RNA PTENP1 and microRNA regulation. Biomaterials 44, 71-81 (2015).

23. Zheng LL, Zhou KR, Liu S et al. DreamBase: DNA modification, RNA regulation and protein binding of expressed pseudogenes in human health and disease. Nucleic Acids Res. 46(D1), D85-D91 (2018).

-• dreamBase: the first comprehensive website tool for analysis of the regulatory features and functions of potentially expressed pseudogenes. 
24. Saeed AI, Sharov V, White J et al. TM4: a free, open-source system for microarray data management and analysis. Biotechniques 34(2), 374-378 (2003).

25. Tang Z, Li C, Kang B et al. GEPIA: a web server for cancer and normal gene expression profiling and interactive analyses. Nucleic Acids Res. 45(W1), W98-W102 (2017).

26. Gyorffy B, Lánczky A, Szállási Z. Implementing an online tool for genome-wide validation of survival-associated biomarkers in ovarian-cancer using microarray data from 1287 patients. Endocr. Relat. Cancer 19(2), 197-208 (2012).

27. Li JH, Liu S, Zhou H et al. starBase v2.0: decoding miRNA-ceRNA, miRNA-ncRNA and protein-RNA interaction networks from large-scale CLIP-Seq data. Nucleic Acids Res. 42(Database issue), D92-D97 (2014).

28. Chou CH, Shrestha S, Yang CD et al. miRTarBase update 2018: a resource for experimentally validated microRNA-target interactions. Nucleic Acids Res. 46(D1), D296-D302 (2018).

29. Shannon P, Markiel A, Ozier O et al. Cytoscape: a software environment for integrated models of biomolecular interaction networks. Genome Res. 13(11), 2498-2504 (2003).

30. Bindea G, Mlecnik B, Hackl H et al. ClueGO: a Cytoscape plug-in to decipher functionally grouped gene ontology and pathway annotation networks. Bioinformatics 25 (8)1091-1093 (2009)

31. Szklarczyk D, Morris JH, Cook H et al. The STRING database in 2017: quality-controlled protein-protein association networks, made broadly accessible. Nucleic Acids Res. 45(D1), D362-D368 (2017).

32. Scardoni G, Petterlini M, Laudanna C. Analyzing biological network parameters with CentiScaPe. Bioinformatics 25(21), 2857-2859 (2009).

33. Chandrashekar DS, Bashel B, Balasubramanya SAH et al. UALCAN: a portal for facilitating tumor subgroup gene expression and survival analyses. Neoplasia 19(8), 649-658 (2017).

34. Uhlén M, Fagerberg L, Hallström BM et al. Proteomics. Tissue-based map of the human proteome. Science 347(6220), 1260419 (2015).

35. Cerami E, Gao J, Dogrusoz U et al. The cBio cancer genomics portal: an open platform for exploring multidimensional cancer genomics data. Cancer Discov. 2(5), 401-404 (2012).

36. Kong Y, Zhang L, Huang Y et al. Pseudogene PDIA3P1 promotes cell proliferation, migration and invasion, and suppresses apoptosis in hepatocellular carcinoma by regulating the p53 pathway. Cancer Lett. 407, 76-83 (2017).

37. Johnson GS, Li J, Beaver LM et al. A functional pseudogene, NMRAL2P, is regulated by Nrf2 and serves as a coactivator of NQO1 in sulforaphane-treated colon cancer cells. Mol. Nutr. Food Res. 61(4), 1600769 (2017).

38. De Martino M, Forzati F, Marfella M et al. HMGA1P7-pseudogene regulates H19 and Igf2 expression by a competitive endogenous RNA mechanism. Sci. Rep. 6, 37622 (2016).

39. De Martino M, Palma G, Azzariti A et al. The HMGA1 pseudogene 7 induces miR-483 and miR-675 upregulation by activating Egr1 through a ceRNA mechanism. Genes (Basel) 8(11) pii:E330 (2017).

40. Esposito F, De Martino M, Forzati F et al. HMGA1-pseudogene overexpression contributes to cancer progression. Cell Cycle 13(23), 3636-3639 (2014).

41. Esposito F, De Martino M, Petti MG et al. HMGA1 pseudogenes as candidate proto-oncogenic competitive endogenous RNAs. Oncotarget 5(18), 8341-8354 (2014).

42. Li G, Wu F, Yang H et al. MiR-9-5p promotes cell growth and metastasis in non-small cell lung cancer through the repression of TGFBR2. Biomed. Pharmacother. 96, 1170-1178 (2017).

43. Drakaki A, Hatziapostolou M, Polytarchou C et al. Functional microRNA high throughput screening reveals miR-9 as a central regulator of liver oncogenesis by affecting the PPARA-CDH1 pathway. BMC Cancer 15, 542 (2015).

44. Zheng L, Qi T, Yang D et al. microRNA-9 suppresses the proliferation, invasion and metastasis of gastric cancer cells through targeting cyclin D1 and Ets1. PLoS ONE 8(1), e55719 (2013).

45. Guo LM, Pu Y, Han Z et al. microRNA-9 inhibits ovarian cancer cell growth through regulation of NF-kappaB1. FEBS J. 276(19), 5537-5546 (2009).

46. Liu D, Li Y, Luo G et al. LncRNA SPRY4-IT1 sponges miR-101-3p to promote proliferation and metastasis of bladder cancer cells through up-regulating EZH2. Cancer Lett. 388, 281-291 (2017).

47. Chang L, Yuan Y, Li C et al. Upregulation of SNHG6 regulates ZEB1 expression by competitively binding miR-101-3p and interacting with UPF1 in hepatocellular carcinoma. Cancer Lett. 383(2), 183-194 (2016).

48. Hou Y, Li L, Ju Y et al. MiR-101-3p regulates the viability of lung squamous carcinoma cells via targeting EZH2. J. Cell. Biochem. 118(10), 3142-3149 (2017).

49. Liu P, Ye F, Xie X et al. mir-101-3p is a key regulator of tumor metabolism in triple negative breast cancer targeting AMPK. Oncotarget 7(23), 35188-35198 (2016).

50. Gregory PA, Bert AG, Paterson EL et al. The miR-200 family and miR-205 regulate epithelial to mesenchymal transition by targeting ZEB1 and SIP1. Nat. Cell Biol. 10(5), 593-601 (2008). 
51. Humphries B, Wang Z, Oom AL et al. microRNA-200b targets protein kinase C $\alpha$ and suppresses triple-negative breast cancer metastasis. Carcinogenesis 35(10), 2254-2263 (2014).

52. Pecot CV, Rupaimoole R, Yang D et al. Tumour angiogenesis regulation by the miR-200 family. Nat. Commun. 4, 2427 (2013).

53. Feng B, Wang R, Song HZ et al. microRNA-200b reverses chemoresistance of docetaxel-resistant human lung adenocarcinoma cells by targeting E2F3. Cancer 118(13), 3365-3376 (2012).

54. Vivanco I, Sawyers CL. The phosphatidylinositol 3-kinase AKT pathway in human cancer. Nat. Rev. Cancer 2(7), 489-501 (2002).

55. Osaki M, Oshimura M, Ito H. PI3K-Akt pathway: its functions and alterations in human cancer. Apoptosis 9(6), 667-676 (2004).

56. Martini M, De Santis MC, Braccini L et al. PI3K/AKT signaling pathway and cancer: an updated review. Ann. Med. 46(6), 372-383 (2014).

57. Kaplan DR, Miller FD. Neurotrophin signal transduction in the nervous system. Curr. Opin. Neurobiol. 10(3), 381-391 (2000).

58. Ricci A, Greco S, Mariotta S et al. Neurotrophins and neurotrophin receptors in human lung cancer. Am. J. Respir. Cell Mol. Biol. 25(4), 439-446 (2001).

59. Meldolesi J. Neurotrophin Trk receptors: new targets for cancer therapy. Rev. Physiol. Biochem. Pharmacol. 174, 67-79 (2018).

60. Louie E, Chen XF, Coomes A et al. Neurotrophin-3 modulates breast cancer cells and the microenvironment to promote the growth of breast cancer brain metastasis. Oncogene 32(35), 4064-4077 (2013).

61. Cui W, Li Y, Xu K et al. miR-361-5p inhibits hepatocellular carcinoma cell proliferation and invasion by targeting VEGFA. Biochem. Biophys. Res. Commun. 479(4), 901-906 (2016).

62. Ghosh A, Dasgupta D, Ghosh A et al. miRNA199a-3p suppresses tumor growth, migration, invasion and angiogenesis in hepatocellular carcinoma by targeting VEGFA, VEGFR1, VEGFR2, HGF and MMP2. Cell Death Dis. 8(3), e2706 (2017).

63. Xu M, Zheng YL, Xie XY et al. Sorafenib blocks the HIF-1 $\alpha$ /VEGFA pathway, inhibits tumor invasion, and induces apoptosis in hepatoma cells. DNA Cell Biol. 33(5), 275-281 (2014). 